\title{
Diversidades filogenética e funcional: novas abordagens para a Ecologia de comunidades
}

\author{
Marcus Vinicius Cianciaruso ${ }^{1,3}$, Igor Aurélio Silva² \& Marco Antônio Batalha ${ }^{2}$ \\ ${ }^{1}$ Departamento de Ecologia, ICB, Universidade Federal de Goiás - UFG, \\ CP 131, CEP 74001-970, Goiânia, GO, Brasil \\ ${ }^{2}$ Departamento de Botânica, Universidade Federal de São Carlos - UFSCar, \\ CP 676, CEP 13565-905, São Carlos, SP, Brasil \\ ${ }^{3}$ Autor para correspondência: Marcus Vinicius Cianciaruso,e-mail: cianciaruso@gmail.com
}

CIANCIARUSO, M.V., SILVA, I.A. \& BATALHA, M.A. Phylogenetic and functional diversities: new approaches to community Ecology. Biota Neotrop. 9(3): http://www.biotaneotropica.org.br/v9n3/en/ abstract?article+bn01309032009.

\begin{abstract}
Although diversity seems to be the most intuitive ecological concept, no consensual definition has been stated. Traditional diversity measures, which take into account only the number of species and their relative contribution, have little predictive power about the functioning of communities. Diversity measures that include information on phylogenetic relationships among species or their functional traits should be better than the traditional measures. We present a short review of the properties and applications of some diversity measures, emphasizing two recent and promising approaches, the phylogenetic and functional diversities, which have been shown to be more sensitive to detect responses of communities to environmental changes. In phylogenetic diversity, species relatedness is taken into account, whereas in functional diversity traits related with community functioning are considered. We also discuss challenges and perspectives for the use of these two approaches in ecology.

Keywords: community, complementarity, diversity, niche, phylogeny.
\end{abstract}

CIANCIARUSO, M.V., SILVA, I.A. \& BATALHA, M.A. Diversidades filogenética e funcional: novas abordagens para a Ecologia de comunidades. Biota Neotrop. 9(3): http://www.biotaneotropica.org.br/v9n3/ pt/abstract?article+bn01309032009.

Resumo: Embora a diversidade pareça ser o conceito ecológico mais intuitivo, nenhuma definição consensual foi formulada. As medidas tradicionais de diversidade, que levam em conta apenas o número de espécies e suas contribuições relativas, têm se mostrado estimativas pouco preditivas da estrutura e do funcionamento das comunidades. Medidas de diversidade que incorporem informações sobre as relações filogenéticas das espécies ou suas características funcionais podem ser melhores do que as medidas tradicionais para muitas finalidades. Apresentamos uma pequena revisão das propriedades e aplicações de algumas medidas de diversidade. Enfatizamos aqui duas abordagens recentes e promissoras, as diversidades filogenética e funcional, que têm se mostrado mais sensíveis para detectar respostas das comunidades às mudanças ambientais. Na diversidade filogenética, as relações de parentesco entre as espécies são levadas em conta, enquanto que na diversidade funcional traços que devem ter relações com o funcionamento das comunidades são considerados. Discutimos ainda os desafios e as perspectivas para o uso dessas duas abordagens na ecologia.

Palavras-chave: complementaridade, comunidade, diversidade, filogenia, nicho. 


\section{Introdução}

Antes mesmo de a ecologia existir como ciência, os naturalistas já estavam interessados nos padrões da distribuição de espécies nas comunidades naturais. Por exemplo, o gradiente latitudinal de riqueza é considerado "o padrão ecológico mais antigo" (Hawkins 2001), já percebido por Humboldt (1808), que escreveu: "Quanto mais perto chegamos dos trópicos, maior o aumento na variedade das estruturas, na beleza das formas e na mistura das cores, assim como na juventude perpétua e no vigor da vida orgânica". Essas primeiras ideias sobre a distribuição das espécies, descritas no século XIX, formaram o embrião da Ecologia como uma ciência única. Não obstante, percepções sobre a distribuição de espécies são mais antigas que nossa própria espécie (Begon et al. 1996). Logo depois que nossos ancestrais adquiriram seus primeiros pensamentos conscientes, eles devem ter percebido o valor de saber onde poderiam achar plantas e animais palatáveis (Worster 1994).

No último século, contudo, nosso conhecimento avançou bastante, e muitas teorias foram formuladas. A teoria da sucessão ecológica (Clements 1916, Gleason 1927), a ubiquidade e a raridade das espécies (Fischer et al. 1943, Preston 1948, MacArthur 1960), a biogeografia de ilhas (MacArthur \& Wilson 1967) e a distribuição latitudinal da diversidade de espécies (Brown 1995) são exemplos de teorias heurísticas na ecologia de comunidades, que contribuíram consideravelmente para a compreensão espácio-temporal dos padrões de diversidade biológica (Rosenzweig 1995, Magurran 2004).

Tais teorias continuam sendo questionadas, outras são ainda formuladas - como a teoria neutra de Hubbell (2001) -, mas questões relacionadas à diversidade biológica não são mais exclusivas à comunidade científica. Nesse sentido, a Conferência Rio-92 foi um divisor de águas, pois, a partir desse encontro, a diversidade biológica deixou de ser assunto de ecólogos e ativistas ambientais, passando a ser uma questão de preocupação pública e debate político (Ricotta 2005). Hoje em dia, muitas pessoas estão conscientes de que a extinção de espécies está se acelerando, ainda que poucas compreendam totalmente a magnitude da perda. Cerca de $20 \%$ da área coberta originalmente com floresta tropical pluvial já foram completamente destruídos, e outros 40\% estão seriamente degradados (Myers 1988). As taxas de extinção podem ser tão altas quanto uma velocidade de perda de três espécies por hora (Wilson 1992).

Tendo em vista esse cenário, questões críticas devem ser respondidas pela ecologia de comunidades. Os problemas ambientais mais urgentes exigem que os cientistas prevejam os efeitos das alterações humanas. Precisamos, por exemplo, prever a densidade e a distribuição de espécies introduzidas artificialmente; avaliar o impacto em uma comunidade da construção de uma rodovia ou determinar as consequências da poluição (Smith 2000). Para isso, a capacidade de previsão das teorias ecológicas tem de melhorar consideravelmente (Peters 1991). No caso das relações entre a diversidade biológica e as funções da comunidade, tais como produtividade, ciclagem de nutrientes, sequestro de carbono e estabilidade, nossa capacidade de previsão ainda é fraca, embora essas relações estejam sendo estudadas há muitas décadas (Ricotta 2005). Essa escassez de resultados inequívocos levou a comentários como o de Hurlbert (1971) sobre o 'não-conceito de diversidade de espécies' e o de Poole (1974) de que medidas de diversidade são 'respostas para questões que ainda não foram encontradas'.

Embora a diversidade pareça o conceito ecológico mais bem estudado e intuitivo, nenhuma definição consensual foi formulada (Ricotta 2005). A inevitável perda de informação que existe quando resumimos um grande conjunto de dados de uma comunidade faz com que alguns digam que os índices tradicionais de diversidade mais escondem do que revelam. Tem-se tornado claro que essas medidas tradicionais são estimativas pouco preditivas da estrutura (Webb 2000, Ricotta et al. 2005) e do funcionamento (Díaz \& Cabido 2001, Petchey 2004) da comunidade. Por exemplo, se uma mudança ambiental qualquer repõe uma comunidade em que as espécies pertencem a gêneros distintos por outra em que a maioria das espécies pertencem ao mesmo gênero, mantendo o mesmo número de espécies e a mesma distribuição de abundâncias, análises tradicionais da diversidade não revelarão nenhum efeito da mudança sobre a comunidade. Da mesma forma, se uma dada mudança ambiental provoca a substituição de uma comunidade em que as espécies têm diferentes vetores de polinização por outra em que as espécies têm um único vetor, mantendo novamente o mesmo número de espécies e a mesma distribuição de abundâncias, então as análises tradicionais também não detectarão nenhuma mudança. Portanto, medidas de diversidade que incorporem informações sobre as relações filogenéticas das espécies (Webb 2000, Ricotta et al. 2005) ou suas características funcionais (Diaz \& Cabido 2001, Petchey \& Gaston 2006) devem ser melhores do que as medidas tradicionais.

Neste artigo, apresentamos uma pequena revisão das propriedades e aplicações de algumas medidas de diversidade. Enfatizamos duas abordagens distintas para medi-la, as diversidades filogenética (Clarke \& Warwick 1998, Webb 2000) e funcional (Petchey \& Gaston 2006), que têm se mostrado mais sensíveis para detectar respostas das comunidades às mudanças ambientais do que as medidas tradicionais de diversidade (Ricotta et al. 2005, Petchey \& Gaston 2006). Sendo assim, aqui nós: i) revemos algumas medidas tradicionais de diversidade; ii) apresentamos a diversidade filogenética, em que as relações de parentesco entre as espécies são levadas em conta; iii) apresentamos a diversidade funcional, em que traços que devem ter relações com o funcionamento das comunidades são considerados; e iv) discutimos os desafios e as perspectivas para o uso dessas duas abordagens promissoras para a Ecologia.

\section{Medidas Tradicionais de Diversidade}

Quando estamos interessados em quantificar e resumir a diversidade de uma comunidade, uma primeira maneira de fazermos isso é simplesmente contar o número de espécies que ali ocorrem. Esse número de espécies pode ser expresso tanto em função de uma unidade de área - e, nesse caso, falamos em "densidade de espécies" - ou de um certo número de indivíduos - e, nesse caso, falamos em "riqueza de espécies" (Krebs 1999). No entanto, o número de indivíduos é sempre contado numa área definida. Portanto, a riqueza continua tendo um componente da densidade de espécies. Quando estimamos a diversidade dessa forma, consideramos tanto uma espécie rara, que aparece com um único indivíduo na comunidade, quanto uma comum, que aparece com um número muito grande de indivíduos, da mesma forma - ambas têm o mesmo peso na quantificação da diversidade estimada pela densidade ou pela riqueza de espécies.

Para evitar esse problema, podemos incluir a contribuição relativa de cada espécie na quantificação. Com isso, chegamos aos chamados índices de diversidade, dos quais muitos foram e continuam sendo propostos, entre os quais os de Shannon e Simpson (Magurran 2004). Se houver $\mathrm{N}$ espécies na comunidade, podemos calcular a contribuição relativa de cada uma delas, medindo algum descritor dos seus indivíduos, como a altura ou a biomassa, ou simplesmente contando o número de indivíduos - o que é o mais comumente feito. A contribuição relativa da i-ésima espécie, em que $\mathrm{i}=1,2, \ldots \mathrm{N}$, pode ser notada como $\mathrm{p}_{\mathrm{i}}$, de modo que $\mathrm{p}_{\mathrm{i}}$ varia de 0 a 1 e a somatória de $\mathrm{p}_{\mathrm{i}}$ é igual a 1. A lógica desses índices é que eles combinam tanto o número de espécies, a riqueza, quanto a equabilidade, a maneira como os indivíduos se distribuem pelas espécies. Ainda que cada índice tenha suas particularidades e cada um pese as espécies raras e 
abundantes de maneiras diferentes, altas riquezas e altas equabilidades estão correlacionadas com altas diversidades (Ricotta 2003).

Da maneira como é usualmente calculada, isto é, pela abundância das espécies, essa contribuição relativa ignora quaisquer outras diferenças entre as espécies. Assim, um indivíduo de jequitibá-rosa (Cariniana legalis (Mart.) Kuntze), que pode chegar a $40 \mathrm{~m}$ de altura e pesar mais de $200 \mathrm{t}$, tem o mesmo peso no cálculo da diversidade que um arbusto na submata, com alguns poucos metros de altura e pesando alguns poucos quilos. Assim, essas medidas tradicionais de diversidade acabam assumindo duas premissas, que são, via de regra, ignoradas pelos pesquisadores. Primeiro, a de que todas as espécies são consideradas equivalentes, isto é, todas têm a mesma importância no que diz respeito à quantidade de informação que carregam, não importando se algumas possuem alto valor de conservação ou ainda se outras são importantes para a manutenção dos processos na comunidade (Magurran 2004). Segundo, dentro de uma dada espécie, assume-se que os indivíduos são equivalentes, isto é, não importa se têm alturas ou biomassas diferentes, se crescem em locais mais ou menos sombreados etc.

Um passo importante na ciência é reconhecer a importância de cada método ou abordagem e decidir ponderadamente quando usar um método ou uma abordagem particular. As medidas tradicionais de diversidade têm respondido às nossas perguntas? Conseguimos chegar a boas previsões com elas? Para ambas as perguntas, as respostas parecem ser negativas. E isso não é surpreendente, já que não só as espécies são diferentes entre si, como indivíduos de uma dada espécie também o são. Nesse caso, medidas que incorporem informações sobre as espécies ou sobre os indivíduos devem ser melhores do que aquelas que não o fazem.

\section{Medidas de Diversidade Filogenética}

A diversidade filogenética é uma medida da diversidade de uma comunidade que incorpora as relações filogenéticas das espécies (Magurran 2004). A premissa principal dessa medida é que a diversidade é maior em uma comunidade em que as espécies são filogeneticamente mais distintas. A percepção dominante em ecologia evolutiva é que espécies coexistindo devem diferir significativamente e que a maior parte da variação entre espécies aparentadas é uma resposta adaptativa à competição no passado, quando os traços não diferiam (Harvey \& Rambaut 2000). A predição geral desse modelo de evolução é que a divergência nos traços ecológicos deve ser ampla. Entretanto, estudos recentes mostraram que muitas linhagens apresentam um conservadorismo dos traços ecológicos dominantes (e.g., Harvey \& Rambaut 2000, Prinzing et al. 2001, Ackerly 2003), isto é, esses traços tendem a se manter ao longo dessas linhagens evolutivas. Por que isso ocorre?

Há várias maneiras pelas quais um ancestral comum recente pode restringir a radiação adaptativa de uma linhagem: i) o tempo para a evolução de diferenças entre táxons filogeneticamente próximos é menor que entre táxons filogeneticamente distantes (inércia filogenética; Felsenstein 1985); ii) padrões arquiteturais, fisiológicos e ontológicos podem enviesar os tipos de traços que podem evoluir (restrições estruturais; Wake \& Larson 1987); e iii) o baixo valor adaptativo de formas transicionais restringe a diversidade de traços que podem evoluir (restrições adaptativas; Wright 1982). Consequentemente, há em geral uma relação positiva entre uma medida da proximidade filogenética entre duas espécies e uma medida de suas histórias de vida e similaridade ecológica (Harvey \& Pagel 1991, Silvertown et al. 1997). Portanto, uma comunidade em que as espécies estão distribuídas em muitos gêneros deve apresentar uma diversidade maior que uma comunidade em que a maioria das espécies pertence a um mesmo gênero (Magurran 2004).
As primeiras tentativas de incorporar a informação filogenética das comunidades em medidas de diversidade remontam à década de 1940. Elton (1946) foi o primeiro a postular que o pequeno número de espécies em gêneros em comunidades locais quando comparadas com o banco regional de espécies era uma evidência da exclusão competitiva entre espécies congenéricas ecologicamente similares. Contudo, somente a partir da década de 1990 é que essa abordagem ganhou força, em consequência de sua importância em estabelecer prioridades na conservação (May 1990). A extinção de uma espécie sem parentes próximos em uma comunidade tende a levar a uma perda maior de informação genética do que a extinção de uma espécie com parentes próximos (May 1990, Williams et al. 1991). Por causa disso, uma melhor estratégia de conservação é estabelecer reservas que contenham a maior diversidade filogenética possível (Vane-Wright et al. 1991, Williams et al. 1991, Faith 1996, mas veja Polasky et al. 2001).

A melhor medida da relação filogenética de duas espécies é a idade do evento de especiação sofrido pelo mais recente ancestral em comum (Harvey \& Pagel 1991). Essa informação pode ser estimada pela diferença nos pares de base de um gene neutro usado em filogenia molecular entre duas espécies (Harvey \& Pagel 1991). Faith (1992) foi o primeiro a propor uma medida de diversidade filogenética baseada na idade da especiação, conhecida como índice PD (do inglês phylogenetic diversity). Essa medida é obtida pela soma dos comprimentos dos braços da árvore filogenética das espécies de uma comunidade (Faith 1992). Longos comprimentos de braços correspondem a tempos evolutivos maiores e, presumivelmente, a grupos taxonômicos mais distintos. Portanto, a PD de uma comunidade é uma função do número de espécies e da diferença filogenética entre elas (Faith 1992). No entanto, o cálculo da PD de grandes comunidades requer que todas as espécies sejam sequenciadas, o que muitas vezes é impraticável. Além disso, não há nenhuma garantia de que a taxa de mudança molecular em um determinado gene seja a mesma em diferentes linhagens de uma comunidade (Webb 2000). Logo, para obter uma boa estimativa da diversidade filogenética da comunidade, é necessário o sequenciamento de vários genes (Webb 2000).

Um método alternativo mais simples é considerar a topologia de uma filogenia. Topologia, em matemática, é o estudo das propriedades das formas geométricas. Considerando as formas geométricas de filogenias, o parentesco entre duas espécies pode ser estimado pela distância topológica entre elas, ou seja, pelo número de nós ou ligações que as separam em uma árvore filogenética (Webb 2000, Ricotta 2004). A ideia foi desenvolvida primeiramente por Vane-Wright et al. (1991) para incorporar um valor taxonômico de cada espécie nas medidas de diversidade. Eles chamaram esse valor específico de distinguibilidade taxonômica (do inglês taxonomic distinctiveness). A distinguibilidade taxonômica de uma espécie $x_{\mathrm{i}}$ em uma dada comunidade é inversamente proporcional ao número de nós ou ligações que ela apresenta em uma árvore filogenética (Vane-Wright et al. 1991). Portanto, uma espécie possuirá uma distinguibilidade maior quanto menos nós ou ligações ela apresentar com outras espécies. Ela também pode ser calculada pela soma das distâncias topológicas (i.e., número de ligações) entre todos os pares de espécies dos quais $x_{\mathrm{i}}$ participa (Figura 1, Ricotta 2004).

Duas medidas promissoras baseadas em distâncias topológicas são a distinção taxonômica ( $\Delta^{*}$, taxonomic distinctness) e a diversidade taxonômica $(\Delta$, taxonomic diversity; Warwick \& Clarke 1995 , 1998, Clarke \& Warwick 1998). Essas medidas são promissoras por serem fáceis de calcular, por terem sido aplicadas em várias comunidades e por serem robustas em relação a diferenças na amostragem (Clarke \& Warwick 1998, Magurran 2004). A diversidade taxonômica é estimada pelo número esperado de nós entre quaisquer dois indivíduos sorteados em uma comunidade (Clarke \& Warwick 1998). A 


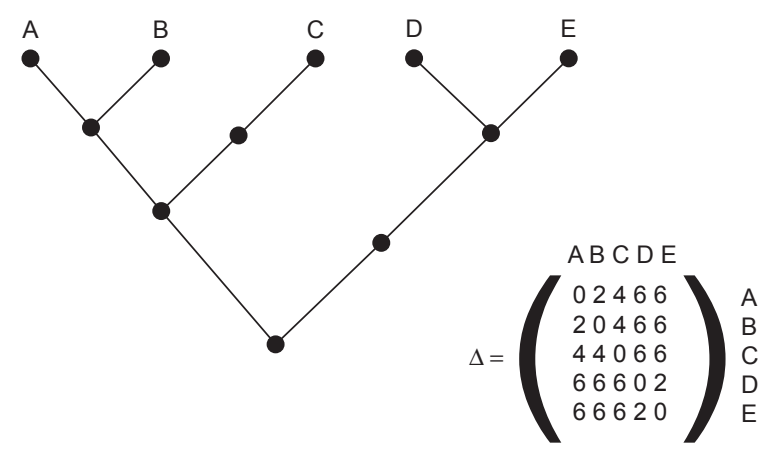

Figura 1. Esquema de uma árvore filogenética com sua matriz de distância. Os valores das distâncias topológicas são o número de ligações entre os pares de espécies. A soma da linha ou coluna de uma espécie é igual ao valor da distinguibilidade taxonômica (modificado de Ricotta 2004).

Figure 1. Phylogenetic tree scheme with the corresponding distance matrix. Topological distance values are the number of links between species pairs. Row or column sum is the taxonomic distinctness value (modified from Ricotta 2004).

distinção taxonômica, por sua vez, é modificada para remover o peso excessivo da abundância das espécies no cálculo da diversidade taxonômica. A distinção taxonômica é estimada pelo número esperado de nós entre quaisquer dois indivíduos de espécies diferentes sorteados em uma comunidade (Clarke \& Warwick 1998). Ambos os índices podem ser calculados usando os programas Past (Hammer et al. 2001) e Primer-E (Clarke \& Warwick 2001a), que estimam intervalos de confiança por aleatorizações.

Os primeiros estudos utilizando a distinção taxonômica e a diversidade taxonômica investigaram as respostas de comunidades bentônicas aos distúrbios antrópicos (Warwick \& Clarke 1995, 1998). Depois disso, essas medidas e suas variações (i.e., variação na distinção taxonômica e distinção taxonômica média; Clarke \& Warwick 2001b) foram usadas para identificar respostas de muitas comunidades ecológicas a vários tipos de distúrbios e gradientes ecológicos (e.g., macrófitos, Mouillot et al. 2005; peixes, Bhat \& Magurran 2006; e plantas, Silva \& Batalha 2006). Recentemente, Ricotta (2004) combinou a abundância relativa das espécies com a distinguibilidade taxonômica em um índice paramétrico de diversidade (sensu Hurlbert 1971). Ele o chamou de distinção taxonômica esperada (expected taxonomic distinctness, Ricotta 2004). A grande vantagem da distinção taxonômica esperada é que podemos variar sua sensibilidade a diferenças na contribuição das espécies raras (Ricotta 2004). Esse índice tem também se apresentado sensível para detectar mudanças na estrutura da comunidade de plantas (Ricotta et al. 2005, Silva \& Batalha 2006).

Outra aplicação interessante é em estudos de interações evolutivas entre hospedeiros e parasitas (Poulin \& Moulliot 2003, 2004, Krasnov et al. 2004). Essas medidas de diversidade têm se mostrado mais sensíveis para identificar padrões de especificidade que as tradicionais. Por exemplo, a distinção taxonômica de pequenos mamíferos parasitados por pulgas foi em geral menor do que o esperado ao acaso, sugerindo que pulgas parasitas usam um espectro taxonômico mais estreito de hospedeiros que o disponível a elas (Krasnov et al. 2004). Estudos da diversidade taxonômica de parasitas de peixes mostraram que populações de Salmonidae adquiriram assembleias de parasitas tão taxonomicamente diversas quanto as populações nativas (Poulin \& Moulliot 2003). Em contraste, estudos anteriores baseados somente na riqueza de espécies encontraram assembleias mais específicas de parasitas em peixes introduzidos do que nos nativos (Kennedy \& Bush 1994).

O desafio da abordagem filogenética em ecologia de comunidades para muitos organismos ainda é desenvolver medidas que incorporem informações derivadas de hipóteses filogenéticas. A distinção e a diversidade taxonômicas, embora robustas, são baseadas na topologia lineana (hierárquica; Warwick \& Clarke 1995, 1998), que em muitas vezes não é uma estimativa acurada da relação filogenética das espécies. Recentemente, filogenias moleculares cladísticas estão sendo publicadas para muitos organismos (e.g., aves, Sibley \& Ahlquist 1990; plantas, Angiosperm Phylogeny Group 2003). Isso permite-nos não só calcular com uma maior facilidade as medidas de diversidade filogenética que considerem o comprimento dos braços (Faith 1992, Webb et al. 2008), como também examinar explicitamente a estrutura filogenética das comunidades (Webb et al. 2002). Nesse sentido, avanços significativos têm sido feitos em ecologia de plantas, de modo que podemos estimar em milhões de anos a diversidade filogenética das comunidades (Webb et al. 2008).

Webb (2000) propôs duas medidas de diversidade baseadas na recente classificação filogenética das angiospermas (Angiosperm Phylogeny Group 2003): a distância média par-a-par (MPD, do inglês mean pairwise distance) e a distância média do vizinho mais próximo (MNND, do inglês mean nearest neighbor distance). A MPD é a distância filogenética média entre todas as combinações de pares de espécies e a MNND é a distância filogenética média do parente mais próximo de todas as espécies (Webb 2000). Enquanto a MPD nos dá um valor geral da estrutura filogenética da comunidade, a MNND é o equivalente às taxas de espécies por gênero (Webb 2000). Essas medidas podem ser calculadas facilmente em dois programas computacionais, o Phylomatic (Webb \& Donoghue 2005) e o Phylocom (Webb et al. 2008). O Phylomatic é uma base de dados e um conjunto de ferramentas para a construção de árvores filogenéticas de angiospermas (Webb \& Donoghue 2005). As árvores geradas são baseadas nas informações de várias filogenias moleculares publicadas (Webb \& Donoghue 2005). Os comprimentos dos braços são baseados nas idades mínimas estimadas para gêneros, famílias e ordens a partir de dados fósseis (Wikström et al. 2001). Os nós não datados são espaçados igualmente entre os nós datados por um algoritmo no Phylocom, chamado BLADJ (do inglês branch length adjustment; Webb et al. 2008). Além disso, o Phylocom calcula várias métricas de estrutura filogenética da comunidade, conservadorismo e correlação de traços (Webb et al. 2008).

Medidas de diversidade filogenética também podem ser usadas para analisar os processos ecológicos que organizam a comunidade (Webb et al. 2002). Comunidades ecológicas são assembleias de espécies coocorrentes que interagem potencialmente uma com a outra. Elas resultam não só de processos ecológicos presentes, como a competição entre as espécies (Hutchinson 1959, Leibold 1998) e os filtros ambientais (Weiher \& Keddy 1995, Chase 2003), mas também de processos evolutivos passados e contínuos (Tofts \& Silvertown 2000, Ackerly 2003). Com base nessas premissas, Webb et al. (2002) propuseram um esquema lógico para inferir mecanismos de coexistência contemporânea (Tabela 1): se os traços funcionais forem conservados na evolução das linhagens de espécies (i.e., traços são mais similares entre as espécies mais próximas filogeneticamente; Ackerly 2003) e os filtros ambientais forem o processo ecológico dominante (Weiher \& Keddy 1995, Webb 2000), níveis de coocorrência entre espécies próximas filogeneticamente tendem a ser altos (atração filogenética). Por outro lado, se os traços funcionais forem filogeneticamente conservados e a competição por recursos limitados for o principal processo ecológico, espécies próximas filogeneticamente tendem a apresentar baixos níveis de coocorrência (dispersão filogenética, Webb et al. 2002), por causa da exclusão competitiva 
Tabela 1. Distribuição filogenética esperada de táxons, considerando as combinações de padrões evolutivos de traços e processos ecológicos (modificado de Webb et al. 2002)

Table 1. Expected phylogenetic distribution of taxons considering the combinations of evolutionary patterns in traits and ecological processes (modified from Webb et al. 2002)

\begin{tabular}{lccc}
\hline $\begin{array}{c}\text { Processo ecológico } \\
\text { dominante: }\end{array}$ & \multicolumn{2}{c}{ Traços ecológicos filogeneticamente } \\
\cline { 2 - 3 } Filtros ambientais & Conservados & & Convergentes \\
\hline Exclusão competitiva & Agregada & & Dispersa \\
\hline
\end{tabular}

de uma ou mais espécies funcionalmente similares (Leibold 1998). No entanto, se os traços funcionais evoluírem convergentemente e as espécies filogeneticamente próximas forem funcionalmente diferentes, os filtros ambientais podem favorecer a coocorrência de espécies filogeneticamente distantes (dispersão filogenética, Webb et al. 2002). A competição, no caso de traços filogeneticamente convergentes, tende a remover qualquer associação sistemática, resultando em comunidades que não são diferentes das esperadas ao acaso.

Para avaliar quais desses processos ecológicos são dominantes em uma comunidade, precisamos: i) testar se os traços são filogeneticamente conservados ou convergentes nas linhagens de plantas; ii) delimitar o banco regional de espécies; iii) calcular as medidas de diversidade filogenética; e iv) comparar as medidas observadas com as obtidas casualmente pelo banco de espécies (Cavender-Bares et al. 2004, 2006).

Há vários métodos para avaliar o padrão de evolução de traços em uma árvore filogenética (e.g., reconstrução com parsimônia, Maddison \& Slatkin 1991; regressão permutacional, Legendre et al. 1994). Um método bastante utilizado é baseado no contraste filogenético independente (Garland et al. 1992). Se as espécies aparentadas forem funcionalmente similares, a magnitude do contraste independente tende a ser similar ao longo da árvore filogenética, resultando em uma pequena variância nos valores dos contrastes (Blomberg et al. 2003). As variâncias observadas são então comparadas às expectativas sob um modelo nulo no qual os valores dos traços são aleatorizados entre as espécies. Se a evolução dos traços for conservada, a variância observada será pequena em relação aos valores esperados sob o modelo nulo. Por outro lado, se a evolução dos traços for convergente, a variância observada será grande em relação aos valores esperados sob o modelo nulo (Blomberg et al. 2003). Essa análise pode ser conduzida no ambiente computacional R (R Development Core Team 2006) com o pacote 'Picante' (Kembel et al. 2008).

O banco regional de espécies é um conjunto de espécies potencialmente capazes de coexistir em uma determinada comunidade (Eriksson 1993). Consequentemente, um banco regional de espécies é ecologicamente delimitado e está relacionado a um tipo de comunidade, a 'comunidade-alvo' (Pärtel et al. 1996). Contudo, o tamanho da comunidade estudada em relação ao do banco regional influencia o poder das análises da estrutura da comunidade (Kraft et al. 2007). Em geral, o maior poder é obtido para comunidades de tamanho intermediário, variando aproximadamente de 30 a $60 \%$ do banco regional (Kraft et al. 2007).

Para calcularmos a MPD e a MNND, precisamos da árvore filogenética do banco regional e de uma lista de espécies da comunidade estudada. A comparação das medidas observadas com a hipótese nula também é feita pelo Phylocom (Webb et al. 2008). Ele faz isso por meio de dois outros índices: o índice de parentesco líquido (NRI, do inglês net relatedness index) e o índice do táxon mais próximo (NTI, do inglês nearest taxon index; Webb, 2000). Valores positivos de NRI indicam que as espécies são mais próximas filogeneticamente do que o esperado ao acaso (Webb 2000). Valores negativos de NRI indicam que as espécies são mais distantes filogeneticamente do que o esperado ao acaso (Webb 2000). O NTI foi proposto como uma medida do agrupamento filogenético terminal em uma filogenia (Webb 2000). Os valores de NTI tendem a ser positivos quando há muitas espécies congenéricas e confamiliares (Webb 2000). Quando isso não ocorre, os valores de NTI tendem a ser negativos (Webb 2000). Também é possível estimar essas medidas de diversidade filogenética com o pacote 'Picante' (Kembel et al. 2008) para o ambiente R (R Development Core Team 2006).

Recentemente, Hardy \& Senterre (2007) propuseram um modelo estatístico para testar a agregação ou a dispersão filogenéticas das comunidades, baseando-se nos coeficientes tradicionais de genética de populações ( $\mathrm{F}_{\mathrm{ST}}$ and $\mathrm{N}_{\mathrm{ST}}$; Hardy et al. 2003). Esse modelo permite uma partição aditiva da diversidade filogenética em um componente alfa e um beta (Hardy \& Senterre 2007). A premissa desse modelo é que a maneira pela qual a diversidade genética é distribuída dada uma genealogia de alelos (Hardy et al. 2003) pode ser usada para descrever a estrutura filogenética das comunidades. Hardy \& Senterre (2007) propuseram dois indices $-\mathrm{I}_{\mathrm{ST}}$ e $\mathrm{P}_{\mathrm{ST}}$ - que representam respectivamente a diversidade de espécies e a diversidade filogenética entre áreas (componente beta). Eles são extensões do clássico índice de diversidade de Simpson (Hardy \& Senterre 2007 para detalhes). Diferenças entre áreas ocorrem quando $\mathrm{I}_{\mathrm{ST}}>0$ e $\mathrm{P}_{\mathrm{ST}}>0$. Quando $\mathrm{P}_{\mathrm{ST}}$ $>\mathrm{I}_{\mathrm{ST}}$, uma agregação filogenética ocorre (Hardy \& Senterre 2007), e os filtros ambientais devem ser o processo ecológico dominante (Webb et al. 2002). Por outro lado, quando $\mathrm{P}_{\mathrm{ST}}<\mathrm{I}_{\mathrm{ST}}$, uma dispersão filogenética ocorre (Hardy \& Senterre 2007), e outros processos ecológicos devem estruturar a comunidade (como a competição; Webb et al. 2002). A razão pela qual isso ocorre é que $I_{S T}$ expressa somente diferenças na frequência de espécies, enquanto $\mathrm{P}_{\mathrm{ST}}$ também expressa a divergência filogenética entre as espécies (Hardy \& Senterre 2007). Uma grande vantagem desse modelo é que é possível estudar os processos ecológicos dominantes entre as comunidades sem conhecer o banco regional de espécies. Além disso, a partição da diversidade filogenética em um componente beta nos permite determinar quais variáveis ambientais estão correlacionadas com a diferença filogenética das comunidades (e.g., altitude; Hardy \& Senterre 2007). É possível estimar esses índices e testar a significância com o progama SPACoDi (Spatial and Phylogenetic Analysis of Community Diversity, Hardy 2007). Para saber detalhes de como testar a significância desses índices, veja Hardy \& Senterre (2007).

Em geral, estudos em diversas comunidades têm encontrado uma agregação filogenética (Vamosi et al. 2009) e, consequentemente, apontam para um papel predominante dos filtros ambientais na estruturação das comunidades (Webb et al. 2002). Isso é particularmente comum em florestas (Webb 2000, Cavender-Bares et al. 2006, Kembel \& Hubbell 2006). Entretanto, o padrão filogenético parece depender da escala espacial. Estudos conduzidos em pequenas escalas têm encontrado uma dispersão filogenética das plantas coocorrentes (Cavender-Bares et al. 2004, 2006, Slingsby \& Verboom 2006). Cavender-Bares et al. (2006) demonstraram, por exemplo, que as espécies de carvalho das florestas temperadas da Flórida são funcionalmente menos similares do que o esperado ao acaso. Embora a floresta de carvalho como um todo apresente uma distribuição agregada de táxons, a competição exclui localmente as espécies funcionalmente similares de carvalho (Cavender-Bares et al. 2006).

Ao contrário das florestas, esperamos uma dispersão filogenética das comunidades de plantas de cerrado (Silva \& Batalha, no prelo). Com base nos modelos de coexistência entre árvores e gramíneas (Sankaran et al. 2004), nas forças ecológicas dependentes da densidade (Becerra 2007, Gilbert \& Webb 2007) e na história evolutiva da 
flora (Pennington et al. 2006), sugerimos que a dispersão filogenética no cerrado seja devida às interações competitivas das plantas, aos ataques de herbívoros e patógenos e à especiação ecológica. Estudos futuros deverão incluir informações sobre a história filogenética dos traços funcionais das plantas do cerrado. Também faltam estudos sobre os processos ecológicos e a estrutura filogenética das plantas em outras formações vegetais. Padrões diferentes do observado em florestas devem também emergir na caatinga por causa da severidade de seus filtros ambientais.

O papel dos filtros ambientais e da competição na determinação das comunidades tem sido dominante na ecologia (Vamosi et al. 2009). Entretanto, outras forças ecológicas como a herbivoria (Becerra 2007) e a facilitação (Valiente-Banuet \& Verdú 2007) podem também gerar comunidades não-aleatórias. A competição é um termo que pode esconder muitos processos ecológicos complexos (Becerra 2007, Gilbert \& Webb 2007) e raramente é testada. Cahill et al. (2008), por exemplo, não encontraram uma relação significativa entre competição e distâncias filogenéticas entre 126 espécies de angiospermas estudadas. Isso sugere que, pelo menos em comunidades de plantas, a dispersão filogenética pode não ser atribuída somente a competição. Além disso, características intraespecíficas também podem determinar a estrutura das comunidades (Johnson \& Stinchcombe 2007). Recentes trabalhos experimentais mostraram que diferenças genotípicas entre os indivíduos e a diversidade genética dentro de populações influenciam a coexistência entre espécies de plantas, a estrutura de comunidades de artrópodes associadas e o funcionamento da comunidade (Johnson \& Stinchcombe 2007). Portanto, estudos futuros devem também considerar a influência de outros fatores na diversidade filogenética das comunidades.

\section{Medidas de Diversidade Funcional}

Assim como no caso da diversidade filogenética, o interesse pela diversidade funcional está crescendo muito nos últimos anos, em diversos campos da Ecologia e em estudos com diversos grupos taxonômicos, sugerindo que o conceito está ganhando importância. Devido à potencial relação entre a diversidade funcional e o funcionamento e manutenção dos processos das comunidades (Petchey \& Gaston 2006), é necessário definir precisamente o conceito de diversidade funcional. Uma definição para diversidade funcional é 'o valor e a variação das espécies e de suas características que influenciam o funcionamento das comunidades' (Tilman 2001). Essa definição é bastante aceita (e.g., Petchey \& Gaston 2002, 2006) e, portanto, é a que adotamos neste trabalho.

Dessa forma, medir a diversidade funcional significa medir a diversidade de características funcionais, que são componentes dos fenótipos dos organismos que influenciam os processos na comunidade. Por exemplo, imaginemos duas comunidades (A e B) com o mesmo número de espécies. Se todas as espécies em A forem dispersas por aves, enquanto que as em B forem dispersas por mamíferos, aves, lagartos e pelo vento, apesar de ambas possuírem o mesmo número de espécies, B será mais diversa por apresentar espécies funcionalmente diferentes no que se refere ao tipo de dispersão. A respeito das características funcionais existe uma vasta literatura, notadamente para as plantas, bem como linhas de pesquisa dedicadas a testar o poder preditivo dessas características em relação a respostas ou efeitos no funcionamento das comunidades e a processos biológicos de difícil mensuração (e.g., Cornelissen et al. 2003, Violle et al. 2007).

A diversidade funcional estima as diferenças entre os organismos diretamente a partir de características funcionais relacionadas com as hipóteses em estudo. Medir a diversidade funcional significa medir a diversidade de traços funcionais que influenciam os processos da comunidade, independentemente da filogenia dos organismos.
Com o crescente interesse por essa abordagem, várias medidas de diversidade funcional estão aparecendo na literatura. Essas medidas diferem na informação que contêm e na maneira com que quantificam a diversidade (Ricotta 2005, Petchey \& Gaston 2006), podendo ser divididas em medidas categóricas ou contínuas.

\section{Medidas categóricas}

A medida de diversidade funcional mais comum, e também a mais antiga, é dada pelo número de grupos funcionais (ou tipos funcionais) presentes em uma comunidade (e.g., Tilman et al. 1997, Díaz \& Cabido 2001), ou seja, a riqueza de grupos funcionais (FGR, do inglês functional group richness). Nessa abordagem, as espécies são agrupadas de acordo com algum método de classificação, de maneira que espécies dentro de um mesmo grupo sejam mais similares entre si do que com espécies de grupos diferentes. Apesar de bastante empregada, a FGR possui algumas limitações importantes. A primeira é que ela necessita de decisões arbitrárias a respeito do nível em que as diferenças entre organismos são funcionalmente significativas. Em outras palavras, quão similares devem ser os organismos para que sejam considerados membros de um mesmo grupo funcional? Essa pergunta geralmente é respondida separando as espécies por algum critério arbitrário, mas existem métodos analíticos que podem reduzir bastante essa subjetividade (e.g., Pillar \& Sosinski 2003). A segunda limitação é que, ao usarmos a FGR, assumimos que: i) membros de um mesmo grupo são funcionalmente idênticos, ou seja, as espécies dentro dos grupos são completamente redundantes; e ii) membros de diferentes grupos são igualmente diferentes, ou seja, complementares. Ambas as assunções raramente são verdadeiras. Portanto, classificar organismos em grupos funcionais requer o maior número de decisões e assunções dentre todas as medidas de diversidade funcional atualmente disponíveis (Petchey \& Gaston 2006). Além disso, a FGR é uma medida categórica e, portanto, menos vantajosa em relação a medidas contínuas (Díaz \& Cabido 2001, Petchey \& Gaston 2006).

\section{Medidas contínuas}

Quando construímos classificações funcionais, podemos estimar a diversidade funcional sem a necessidade de dividir os organismos em grupos arbitrários. Essencialmente, essa abordagem consiste em medir a dispersão de pontos (espécies) em um espaço n-dimensional de características funcionais de modo que as expectativas teóricas de como as medidas de diversidade devem se comportar sejam atendidas (Ricotta 2005, Petchey \& Gaston 2006). Por exemplo, é intuitivo esperar que a adição de uma espécie funcionalmente similar a uma outra já existente não altere significativamente a diversidade funcional e que a adição de uma espécie funcionalmente diferente resulte no seu aumento (e.g., Mason et al. 2003, Petchey \& Gaston 2006). Como as medidas contínuas são vantajosas em relação às categóricas (Díaz \& Cabido 2001, Petchey \& Gaston 2002, 2006), é preferível calcular a diversidade funcional diretamente dos caracteres das espécies (Petchey \& Gaston 2002, Mason et al. 2003). Atualmente, as medidas de diversidade funcional que se enquadram no requisito acima são basicamente de dois tipos: medidas que permitem o uso de apenas uma característica funcional (e.g., Mason et al. 2003, Mason et al. 2005) e medidas multivariadas, que permitem o uso de várias características simultaneamente. As últimas ainda podem ser divididas em medidas baseadas na distância de pares de espécies (e.g., Walker et al. 1999, Ricotta 2005), no "volume" que as espécies de uma comunidade ocupam em um espaço multivariado (Cornwell et al. 2006) ou em uma medida baseada nos fundamentos da análise de agrupamento (Petchey \& Gaston 2002). Discutiremos aqui apenas as medidas multivariadas, pois elas são certamente as mais promissoras. 


\section{Medidas da distância de pares de espécies}

Podemos imaginar as espécies como pontos dispersos em um espaço de $\mathrm{n}$ dimensões, onde $\mathrm{n}$ é igual ao número de características funcionais, e que existem linhas conectando os pares de espécies. Se tivermos s espécies, então teremos $\left(\mathrm{s}^{2}-\mathrm{s}\right) / 2$ linhas, e o comprimento dessas linhas será a distância entre os pares de espécies. Várias medidas de diversidade funcional quantificam essas distâncias. Walker et al. (1999) foram os primeiros a propor uma medida obtida diretamente das características funcionais das espécies (FAD, do inglês functional attribute diversity). A FAD estima a dispersão pela soma das distâncias pareadas entre as espécies no espaço multidimensional, enquanto que uma medida semelhante o faz pela média dessas distâncias (Heemsbergen et al. 2004). Outra medida proposta recentemente baseia-se na entropia quadrática de Rao (Rao 1982) e é semelhante às anteriores, mas permite a inclusão da abundância das espécies. Essas medidas têm a vantagem de serem matematicamente simples e bastante utilizadas (Rao 1982, Botta-Dukát 2005, Ricotta 2005).

Porém, essas medidas apresentam duas propriedades indesejáveis para medidas de diversidade: i) por razões matemáticas, discutidas em Ricotta (2005) e Petchey \& Gaston (2006), essas medidas violam o princípio da monotonicidade, ou seja, o seu valor pode diminuir com a inclusão ou aumentar com a exclusão de espécies, o que a principio é contra-intuitivo; e ii) essas medidas consideram, erroneamente, que as distâncias entre os pares de espécies são independentes, levando a uma estimativa inflacionada da diversidade funcional (Petchey \& Gaston 2006). Apesar dessas deficiências, muitos trabalhos utilizam essas medidas, seduzidos especialmente pela capacidade de incorporar a abundância das espécies. Dessa forma, é necessário cautela na interpretação desses índices. Lepš et al. (2006) disponibilizaram uma macro para o programa Excel (Microsoft 2007) que permite calcular os índices de diversidade de Rao (1982) e o índice de divergência funcional proposto por Mason et al. (2005). Essa macro pode ser obtida em http://botanika.bf.jcu.cz/suspa/FunctDiv/InstrFunctDiv. pdf. Todavia, uma maneira eficaz de ponderar a diversidade funcional pela a abundância das espécies ainda é um desafio em aberto.

\section{Soma do comprimento dos braços do dendrograma}

Petchey \& Gaston $(2002,2006)$ apresentaram uma medida de diversidade funcional (FD, do inglês functional diversity). A FD consiste na soma dos comprimentos dos braços de um dendrograma funcional, ou seja, um dendrograma gerado a partir de uma matriz de "espécies $\times$ características funcionais". O uso de um dendrograma requer mais decisões a serem tomadas a priori; por exemplo, qual a medida de distância e método de ligação que será usado no agrupamento hierárquico. Porém, quando mensurada dessa maneira a diversidade funcional não diminui se uma espécie for adicionada à comunidade, nem aumenta se uma espécie for removida, além de não se alterar quando uma espécie idêntica a outra já existente é adicionada ou removida (Petchey \& Gaston 2006). Além disso, o uso do dendrograma leva em conta a dependência das distâncias entre as espécies no espaço n-dimensional, evitando a inflação da medida como no caso da FAD. Como uma quantificação da diversidade funcional, a FD mede a extensão da complementaridade entre os valores de características das espécies (Petchey \& Gaston 2002) e, portanto, está diretamente ligada ao conceito de nicho ecológico. Diferenças maiores entre esses valores representam maior complementaridade e, portanto, maior FD (Petchey \& Gaston 2002). Como essa medida não considera as abundâncias das espécies, podemos considerá-la mais apropriadamente como uma medida de "riqueza funcional".

O cálculo da FD é relativamente simples e baseia-se em fundamentos da análise de agrupamento. Existem quatro passos para o seu cálculo: i) obter uma matriz funcional (espécies $\times$ características fun- cionais), ii) converter a matriz funcional em uma matriz de distância, iii) realizar o agrupamento da matriz de distância para produzir um dendrograma, e iv) calcular o comprimento total das ramificações do dendrograma (Figura 2). Usualmente a FD é calculada por meio da distância euclidiana e do método de agrupamento pela média, padronizando todas as características de maneira que tenham média igual a zero e variância igual a um. Aparentemente, a FD é pouco afetada pela distância utilizada (Petchey \& Gaston 2006, 2007). No entanto, quando características contínuas (e.g. massa do corpo, altura) e categóricas (e.g., dieta, tipo de dispersão) forem utilizadas simultâneamente recomenda-se o uso da distância de Gower ao invés da distância Euclidiana (Podani \& Schmera 2006). A FD pode ser calculada no ambiente R (R Development Core Team 2006), utilizando as linhas de comando disponíveis na página pessoal de Owen L. Petchey (http://owenpetchey.staff.shef.ac.uk/Code/Code/calculatingfd.html). Ainda é possível encontrar o código para calcular a FD e outras medidas de diversidade funcional na página pessoal de Evan Weiher (http://www.uwec.edu/weiher/weiher_R.htm). Finalmente, existe um pacote desenvolvido por Etienne Laliberté que permite calcular e analisar uma série de medidas de diversidade funcional, a maioria apresentadas aqui, e que pode ser obtido em http://cran.rproject.org/web/packages/FD/.

Petchey et al. (2004) compararam quatro medidas de diversidade - riqueza de espécies, FGR, FAD e FD - e concluíram que as últimas explicam melhor as diferenças funcionais da comunidade. A $\mathrm{FD}$, ao contrário da FAD, não é afetada pela riqueza de espécies per se (Petchey et al. 2004). Uma vez que uma medida de diversidade funcional que não é influenciada pelo número de espécies é um melhor previsor do funcionamento de uma comunidade, a FD tem um poder explanatório maior do que a FAD (Petchey et al. 2004). Até o momento, a FD se mostrou a medida de diversidade funcional que melhor se relaciona com o funcionamento das comunidades (Petchey \& Gaston 2006). Uma outra medida que se propõe a quantificar o espaço ocupado pelas espécies em um espaço multivariado definido pelas características funcionais é o chamado convex hull volume (algo como volume de cápsula convexa, Villéger et al. 2008). Nessa medida, a diversidade funcional seria igual ao volume dessa cápsula e a sua superfície delimitada pelas espécies mais complementares (i.e., aquelas funcionalmente mais distintas). Porém, essa abordagem traz

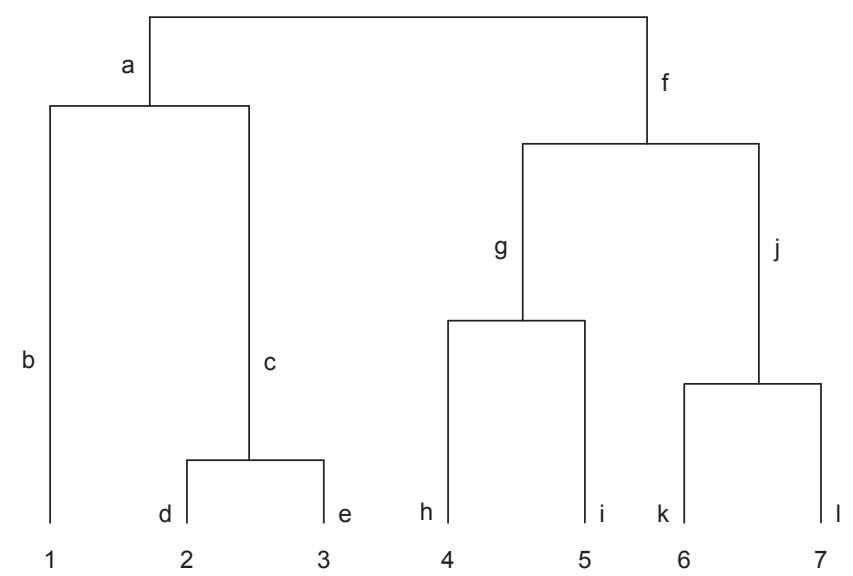

Figura 2. Dendrograma funcional hipotético. A diversidade funcional (FD) é igual à soma de todos os braços necessários para conectar as espécies presentes em uma dada comunidade. Por exemplo, uma comunidade formada pelas espécies 1, 2, 5 e 6 terá uma FD igual $a b+a+c+d+f+g+i+j+k$.

Figure 2. Hypothetical functional dendrogram. Functional diversity (FD) corresponds to the sum of all branches which are necessary to connect all species within a given community. For example, a community with species 1, 2, 5, and 6 will have a FD value equal to $b+a+c+d+f+g+i+j+k$. 
alguns problemas importantes: i) para o volume seja diferente de zero é necessário que o número de espécies seja, pelo menos, uma unidade maior que o número de traços funcionais utilizados. Portanto, comunidades com poucas espécies podem não ter sua diversidade funcional calculada por esse métodos e ii) as espécies contidas dentro da cápsula não contribuem para a diversidade funcional, pois esta é determinada apenas pelas espécies que formam a superfície da cápsula. Assim, não importa o número de espécies, e suas identidades funcionais, se elas estiverem contidas dentro do volume. De todo modo, as funções para o cálculo dessa medida se encontram em http://www.ecolag. univ-montp2.fr/software/F_RED.R.

\section{Aplicações}

Como vimos, a diversidade funcional pode nos ajudar a esclarecer os processos que determinam o funcionamento das comunidades, pois é um conceito que liga organismos e comunidades por meio de mecanismos como complementaridade no uso de recursos e facilitação (Petchey \& Gaston 2006). Questões como "regras de assembleia", "gradientes de diversidade (funcional)" e até mesmo a "teoria neutra" (Hubbell 2001) podem ser investigadas com essa abordagem. Podemos, por exemplo, testar se os filtros ambientais ou a competição são processos determinantes na organização das comunidades. Nesse caso, não precisamos investigar a evolução dos traços ou o parentesco das espécies, pois estamos usando diretamente os traços funcionais de interesse. Se uma determinada comunidade possuir menor FD do que o esperado ao acaso, podemos inferir que filtros ambientais selecionam espécies funcionalmente mais similares; se encontrarmos o oposto (maior FD do que o esperado ao acaso), podemos inferir que a competição é um processo importante (Weiher \& Keddy 1995). Ainda, podemos avaliar com maior precisão as implicações da extinção de espécies na manutenção das características das comunidades (Petchey \& Gaston 2006). Qual o número de extinções que uma comunidade pode suportar antes que funções importantes sejam perdidas? Além disso, a diversidade funcional mostrou-se mais interessante na avaliação e monitoramento de impactos ambientais quando comparada com a riqueza de espécies (Ernst et al. 2006). Finalmente, essa abordagem não depende da identificação taxonômica dos organismos, o que é bastante interessante para regiões onde o conhecimento florístico é ainda deficiente, como, por exemplo, nos trópicos (Duckworth et al. 2000).

Recentemente, uma modificação da FD, chamada iFD (Cianciaruso et al. 2009), permite incluir também a variação intraespecífica das espécies presentes na comunidade. Nesse caso, substitui-se a matriz funcional de 'espécies $\times$ características funcionais' por uma matriz de 'indivíduos $\times$ características funcionais'. A inclusão da variabilidade intraespecífica justifica-se, pois existem fortes evidências de que as diferenças funcionais entre indivíduos de uma mesma espécie são importantes para os processos da comunidade. A variabilidade intraespecífica influencia a habilidade competitiva e coexistência das espécies (Begon \& Wall 1987, Callaway et al. 2003), a invasibilidade (Sexton et al. 2001) e o funcionamento das comunidades, por exemplo, a ciclagem de nutrientes (Madritch \& Hunter 2003), resistência a perturbações (Reusch et al. 2005) e produtividade (Norberg et al. 2001). Portanto, espera-se que a iFD permita relacionar com maior eficiência os organismos presentes na comunidade com os processos ecológicos e variáveis ambientais (Pachepsky et al. 2007).

\section{Conclusões}

Procuramos apresentar duas visões bastante promissoras para a solução de diversos paradigmas ecológicos, mas que ainda são pouco aplicadas por pesquisadores brasileiros. Como há muitas questões sem resposta, este é um momento bastante apropriado para que os pesquisa- dores brasileiros contribuam significativamente para o avanço da Ecologia. Estamos em um dos países com maior biodiversidade no mundo (Mittermeier et al. 2005), de proporções continentais e com comunidades naturais ainda pouco compreendidas, onde podemos tentar responder a muitas das questões apresentadas aqui. Evidentemente, ainda existem grandes lacunas a respeito do conhecimento que temos sobre essa biodiversidade, especialmente sobre os aspectos funcionais das espécies que, muitas vezes, é praticamente inexistente. Porém, acreditamos que isso deva servir como estímulo às pesquisas nessa área e na criação de linhas de financiamento que contemplem a criação de um banco de dados com informações filogenéticas e funcionais sobre nossas espécies.

\section{Agradecimentos}

M.V.C agradece à Fapesp pela bolsa de doutorado e à Capes pela bolsa de doutorado-sanduíche; I.A.S agradece ao CNPq pela bolsa de doutorado; e M.A.B agradece o auxílio à pesquisa concedido pela Fapesp e à bolsa de produtividade em pesquisa concedida pelo CNPq. Agradecemos também as sugestões e comentários feitos pelo Dr. Fernando Roberto Martins.

\section{Referências Bibliográficas}

ACKERLY, D.D. 2003. Community assembly, niche conservatism and adaptive evolution in changing environments. Int. J. Plant. Sci. 164(3 Suppl.):S165-S184.

Angiosperm Phylogeny Group - APG. 2003. An update of the Angiosperm Phylogeny Group classification for the orders and families of flowering plants: APG II. Bot. J. Linn. Soc. 41:399-436.

BECERRA, J.X. 2007. The impact of herbivore-plant coevolution on plant community structure. P. Natl. Acad. Sci. 104(18):7483-7488.

BEGON, M. \& WALL, R. 1987. Individual variation and competitor coexistence: a model. Funct. Ecol. 1(3):237-241.

BEGON, M., HARPER, J.L. \& TOWNSEND, C.R. 1996. Ecology: individuals, populations and communities. Blackwell, Oxford, p.1101.

BHAT, A. \& MAGURRAN, A.E. 2006. Taxonomic distinctness in a linear system: a test using a tropical freshwater fish assemblage. Ecography. 29(1):104-110.

BLOMBERG, S.P., GARLAND Jr., T. \& IVES, A.R. 2003. Testing for phylogenetic signal in comparative data: behavioral traits are more labile. Evolution. 57(4):717-745.

BOTTA-DUKÁT, Z. 2005. Rao's quadratic entropy as a measure of functional diversity based on multiple traits. J. Veg. Sci. 16:533-540.

BROWN, J.H. 1995. Macroecology. University of Chicago, Chicago, p. 269.

CAHILL Jr., J.F., KEMBEL, S.W., LAMB, E.G. \& KEDDY, P.A. 2008. Does phylogenetic relatedness influence the strength of competition among vascular plants? Perspect. Plant. Ecol. 10(1):41-50.

CALLAWAY, R.M., PENNINGS, S.C. \& RICHARDS, C.L. 2003. Phenotypic plasticity and interactions among plants. Ecology. 84(5):1115-1128.

CAVENDER-BARES, J., ACKERLY, D.D., BAUM, D.A. \& BAZZAZ, F.A. 2004. Phylogenetic overdispersion in Floridian oak communities. Am. Nat. 163(6):823-843.

CAVENDER-BARES, J., KEEN, A. \& MILES, B. 2006. Phylogenetic structure of Floridian plant communities depends on taxonomic and spatial scale. Ecology. 87(S7):S109-S122.

CIANCIARUSO, M.V., BATALHA, M.A., GASTON, K.J. \& PETCHEY, O.L. 2009. Including intraspecific variability in functional diversity. Ecology. 90(1):81-89.

CHASE, J.M. 2003. Community assembly: when should history matter? Oecologia. 136(4):489-498.

CLARKE, K.R. \& WARWICK, R.M. 1998. A taxonomic distinctness index and its statistical properties. J. Appl. Ecol. 35(4):523-531. 
CLARKE, K.R. \& WARWICK, R.M. 2001a. Change in marine communities: an approach to statistical analysis and interpretation, Primer-E. Plymouth Marine Laboratory, Plymouth. www.pml.ac.uk/primer/ (último acesso em 28/08/2009).

CLARKE, K.R. \& WARWICK, R.M. 2001b. A further biodiversity index applicable to species lists: variation in taxonomic distinctness. Mar. Ecol. Prog. Ser. 216:265-278.

CLEMENTS, F.E. 1916. Plant succession: an analysis of the development of vegetation. Carnegie Institution of Washington, Washington, p.512.

CORNELISSEN, J.H.C., LAVOREL, S., GARNIEL, E., DÍAZ, S., BUCHMANN, N., GUREVICH, D.E., REICH, P.B., Ter STEEGE, H., MORGAN, H.D., Van der HEIJDEN, M.G.A., PAUSAS, J.G. \& POORTER, H. 2003. A handbook of protocols for standardised and easy measurement of plant functional traits worldwide. Aust. J. Bot. 51:335-380.

CORNWELL, W.K., SCHWILK, D.W. \& ACKERLY, D.D. 2006. A trait-based test for habitat filtering: convex hull volume. Ecology. 87(6):1465-1471.

DIAZ, S. \& CABIDO, M. 2001. Vive la différence: plant functional diversity matters to ecosystem processes. Trends Ecol. Evol. 16(8):646-655.

DUCKWORTH, J.C., KENT, M. \& RAMSAY, P.M. 2000. Plant functional types: an alternative to taxonomic plant community description in biogeography? Prog. Phys. Geog. 24(4):515-542.

ELTON, C. 1946. Competition and the structure of ecological communities. J. Anim. Ecol. 15(1):54-68.

ERIKSSON, O. 1993. The species-pool hypothesis and plant community diversity. Oikos. 68(2):371-374.

ERNST, R., LINSENMAIR, K.E. \& RODEL, M.O. 2006. Diversity erosion beyond the species level: dramatic loss of functional diversity after selective logging in two tropical amphibian communities. Biol. Conserv. 133(2):143-155.

FAITH, D.P. 1992. Conservation evaluation and phylogenetic diversity. Biol. Conserv. 61(1):1-10.

FAITH, D.P. 1996. Conservation priorities and phylogenetic pattern. Conserv. Biol. 10(4):1286-1289.

FELSENSTEIN, J. 1985. Phylogenies and the comparative method. Am. Nat. 125(1):1-15.

FISCHER, R.A., CORBERT, A.S. \& WILLIAMS, C.B. 1943. The relation between the number of species and the number of individuals in a random sample of an animal population. J. Anim. Ecol. 12(1):42-58.

GARLAND Jr., T., HARVEY, P.H. \& IVES, A.R. 1992. Procedures for the analysis of comparative data using phylogenetically independent contrasts. Syst. Biol. 41(1):18-32.

GLEASON, H.A. 1927. Further views on the succession-concept. Ecology. 8(3):299-326.

GILBERT, G.S. \& WEBB, C.O. 2007. Phylogenetic signal in plant pathogenhost range. P. Natl. Acad. Sci. 104:4979-4983.

HAMMER, Ø., HARPER, D.A.T. \& RYAN, P.D. 2001. Past: paleontological statistics software package for education and data analysis. Palaeontologia Electronica. 4(1):1-9. http://palaeo-electronica.org/2001-1/past/issue101.htm (último acesso em 28/08/2009).

HARDY, O.J. 2007. Spacodi: spatial and phylogenetic analysis of community diversity. Université Libre de Bruxeles, Bruxeles. http://www.ulb.ac.be/ sciences/bioancel/ohardy/index.html. (último acesso em 28/08/2009).

HARDY, O.J., CHARBONNEL, N., FRÉVILLE, H. \& HEUERTZ, M. 2003 Microsatellite allele sizes: a simple test to assess their significance on genetic differentiation. Genetics. 163:1467-1482.

HARDY, O.J. \& SENTERRE, B. 2007. Characterizing the phylogenetic structure of communities by an additive partitioning of phylogenetic diversity. J. Ecol. 95:403-506.

HARVEY, P.H. \& PAGEL, M.D. 1991. The comparative method in evolutionary biology. Oxford University, Oxford, p.239.

HARVEY, P.H. \& RAMBAUT, A. 2000. Comparative analyses for adaptive radiations. Phil. Trans. R. Soc. Lond. B. 355:1599-1606.
HAWKINS, B.A. 2001. Ecology's oldest pattern? Trends Ecol. Evol. $16(8): 470$.

HEEMSBERGEN, D.A., BERG, M.P., LOREAU, M., Van HAL, J.R., FABER, J.H. \& VERHOEF, H.A. 2004. Biodiversity effects on soil processes explained by interspecific functional dissimilarity. Science. 306(5698):1019-1020.

HUBBELL, S.P. 2001. The unified neutral theory of biodiversity and biogeography. Princeton University, Princeton, p.448.

HUMBOLDT, A.F. von. 1808. Ansichten der Natur. http://gutenberg. spiegel.de/?id=5\&xid=3736\&kapitel=1\#gb_found (último acesso em 28/08/2009).

HURLBERT, S.H. 1971. The nonconcept of species diversity: a critique and alternative parameters. Ecology. 52(4):577-586.

HUTCHINSON, G.E. 1959. Homage to Santa Rosalia, or why there are so many kinds of animals? Am. Nat. 93(870):145-159.

JOHNSON, M.T.J. \& STINCHCOMBE, J.R. 2007. An emerging synthesis between community ecology and evolutionary biology. Trends Ecol. Evol. 22(5):250-256

KEMBEL, S.W. \& HUBBELL, S.P. 2006. The phylogenetic structure of a neotropical forest tree community. Ecology. 87(7):S86-S99.

KEMBEL, S., ACKERLY, D., BLOMBERG, S., COWAN, P., HELMUS, M. \& WEBB, C. 2008 Picante: tools for integrating phylogenies and ecology. (R package, version 0.2-0). http://picante.r-forge.r-project.org/ (último acesso em 28/08/2009).

KENNEDY, C.R. \& BUSH, A.O. 1994. The relationship between pattern and scale in parasite communities: a stranger in a strange land. Parasitology. 109(2):187-196.

KRAFT, N.J.B., CORNWELL, W.K., WEBB, C.O. \& ACKERLY, D.D. 2007. Trait evolution, community assembly, and the phylogenetic structure of ecological communities. Am. Nat. 170(2):271-283.

KRASNOV, B.R., MOUILLOT, D., SHENBROT, G.I., KHOKHLOVA, I.S. \& POULIN, R. 2004. Geographical variation in host specificity of fleas (Siphonaptera) parasitic on small mammals: the influence of phylogeny and local environmental conditions. Ecography. 27(6):787-797.

KREBS, C.J. 1999. Ecological methodology. Harper \& Row, New York, p. 624.

LEGENDRE, P., LAPOINTE, F. \& CASGRAIN, P. 1994. Modeling brain evolution from behavior: a permutational regression approach. Evolution. 48(55):1487-1499.

LEIBOLD, M. 1998. Similarity and local coexistence of species in regional biotas. Evol. Ecol. 12(1):95-100.

LEPŠ, J., de BELLO, F., LAVOREL, S. \& BERMAN, S. 2006. Quantifying and interpreting functional diversity of natural communities: practical considerations matter. Preslia. 78:481-501.

MACARTHUR, R.H. 1960. On the relative abundance of species. Am. Nat. 94(1):25-36.

MACARTHUR, R.H. \& WILSON, E.O. 1967. The theory of island biogeography. Princeton University, Princeton, p. 224.

MADDISON, W.P. \& SLATKIN, M. 1991. Null model for the number of evolutionary steps in a character on a phylogenetic tree. Evolution. 45(5):1184-1197.

MADRITCH, M.D. \& HUNTER, M.D. 2003. Intraspecific litter diversity and nitrogen deposition affect nutrient dynamics and soil respiration. Oecologia. 136(1):124-128.

MAGURRAN, A.E. 2004. Measuring biological diversity. Blackwell, Oxford, p. 256.

MASON, N.W.H., MACGILLIVRAY, K., STEEL, J.B. \& WILSON, J.B. 2003. An index of functional diversity. J. Veg. Sci. 14(4):571-578.

MASON, N.W.H., MOUILLOT, D., LEE, W.G. \& WILSON, J.B. 2005. Functional richness, functional eveness and functional divergence: the primary components of functional diversity. Oikos. 111(1):112-118.

MAY, R.M. 1990. Taxonomy as destiny. Nature. 347:129-130.

Microsoft Corporation. 2007. Microsoft Excel. Microsoft Corporation, Redmond. 
MITTERMEIER, A.R., FONSECA, G.A.B., RYLANDS, A.B. \& BRANDON, K. 2005. A brief history of biodiversity conservation in Brazil. Conserv. Biol. 19: 601-607.

MOUILlOT, D., GAILlARD, S., ALIAUMEA, C., VERLAQUE, M., BELSHER, T., TROUSSELLIER, M. \& CHI, T.D. 2005. Ability of taxonomic diversity indices to discriminate coastal lagoon environments based on macrophyte communities. Ecol. Indic. 5(1):1-17.

MYERS, N. 1988. Tropical forests and their species: going, going...? In Biodiversity (E.O. Wilson, ed.). National Academy, Washington, p. 28-35.

NORBERG, J., SWANEY, D.P., DUSHOFF, J., LIN, J., CASAGRANDI, R. \& LEVIN, S. A. 2001. Phenotypic diversity and ecosystem functioning in changing environments: a theoretical framework. P. Natl. Acad. Sci. 98(20):11376-11381.

PACHEPSKY, E., BOWN, J.L., EBERST, A., BAUSENWEIN, U., MILLARD, P., SQUIRE, G.R., SQUIRE, G. \& CRAWFORD, J.W. 2007. Consequences of intraspecific variation for the structure and function of ecological communities Part 2, Linking diversity and function Ecol. Model. 207(2):277-285.

PÄRTEL, M., ZOBEL, M., ZOBEL, K. \& Van der MAAREL, E. 1996. The species pool and its relation to species richness: evidence from Estonian plant communities. Oikos. 75(1):111-117.

PENNINGTON, R.T., RICHARDSON, J.E. \& LAVIN, M. 2006. Insights into the historical construction of species-rich biomes from dated plant phylogenies, neutral ecological theory and phylogenetic community structure. New Phytol. 172(4):605-616.

PETCHEY, O.L. 2004. On the statistical significance of functional diversity. Funct. Ecol. 18(2): 297-303.

PETCHEY, O.L. \& GASTON, K.J. 2002. Functional Diversity (FD), species richness, and community composition. Ecol. Lett. 5(3):402-411.

PETCHEY, O.L. \& GASTON, K.J. 2006. Functional diversity: back to basics and looking forward. Ecol. Lett. 9(6):741-758.

PETCHEY, O.L., HECTOR, A. \& GASTON, K.J. 2004. How do measures of functional diversity perform? Ecology. 85(3):847-857.

PETCHEY, O.L. \& GASTON, K.J. 2007. Dendrograms and measuring functional diversity. Oikos. 116(8):1422-1426.

PETERS, R.H. 1991. A critique for ecology. Cambridge University, Cambridge. PILLAR, V.P. \& SOSINSKI Jr., E.E. 2003. An improved method for searching plant functional types by numerical analysis. J. Veg. Sci. 14(3):323-332.

PODANI, J. \& SCHMERA, D. 2006. On dendrogram-based measures of functional diversity. Oikos. 115(1):179-185.

POLASKY, S., CSUTI, B., VOSSLER, C.A. \& MEYERS, S.M. 2001. A comparison of taxonomic distinctness versus richness as criteria for setting conservation priorities for North American birds. Biol. Conserv. 97(1):99-105

POOLE, R.W. 1974. Introduction to quantitative ecology. McGraw-Hill, New York, p. 532.

POULIN, R. \& MOUILLOT, D. 2003. Parasite specialisation from a phylogenetic perspective: a new index of host specificity. Parasitology. 126(5):473-480.

PRESTON, F.W. 1948. The commonness, and rarity, of species. Ecology. 29(1):254-283.

PRINZING, A., DURKA, W., KLOTZ, S. \& BRANDL, R. 2001. The niche of higher plants: evidence for phylogenetic conservatism. P. Roy. Soc. B - Biol. Sci. 268:2383-2389.

R Development Core Team. 2006. R: a language and environment for statistical computing. R Foundation for Statistical Computing, Vienna. http:// www.R-project.org (último acesso em 28/08/2009).

RAO, C.R. 1982. Diversity and dissimilarity coefficients: a unified approach. Theor Popul. Biol. 21(1):24-43.

REUSCH, T.B.H., EHLERS, A., HAMMERLI, A. \& WORM, B. 2005. Ecosystem recovery after climatic extremes enhanced by genotypic diversity. P. Natl. Acad. Sci. 102:2826-2831.
RICOTTA, C. 2003. On parametric evenness measures. J. Theor. Biol. 222(1):189-197.

RICOTTA, C. 2004. A parametric diversity measure combining the relative abundances and taxonomic distinctiveness of species. Divers. Distrib. 10(1):143-146.

RICOTTA, C. 2005. Through the jungle of biological diversity. Acta Biotheor. 53(1):29-38.

RICOTTA, C., AVENA, G. \& CHIARUCCI, A. 2005. Quantifying the effects of nutrient addition on the taxonomic distinctness of serpentine vegetation. Plant. Ecol. 179(1):21-29.

ROSENZWEIG, M.L. 1995. Species diversity in space and time. Cambridge University, Cambridge, p. 436.

SANKARAN, M., RATNAM, J. \& HANAN, N.P. 2004. Tree-grass coexistence in savannas revisited: insights from an examination of assumptions and mechanisms invoked in existing models. Ecol. Lett. 7(6):480-490.

SEXTON, J.P., MCKAY, J.K. \& SALA, A. 2001. Plasticity and the genetic diversity may allow saltcedar to invade cold climates in North America. Ecol. Appl. 12(6):1652-1660.

SIBLEY, C.G. \& AHLQUIST, J.E. 1990. Phylogeny and classification of birds: a study in molecular evolution. Yale University, New Haven, p. 1080.

SILVA, I.A. \& BATALHA, M.A. 2006. Taxonomic distinctness and diversity of a hyperseasonal savanna in central Brazil. Divers. Distrib. 12(6):725-730.

SILVA, I.A. \& BATALHA, M.A. 2009. Phylogenetic overdispersion of plant species in southern Brazilian savannas. Braz. J. Biol. 69(2):631-637.

SILVERTOWN, J., FRANCO, M. \& HARPER J.L. 1997. Plant life histories: ecology, phylogeny and evolution. Cambridge University, New York, p.313.

SLINGSBY, J.A. \& VERBOOM, G.A. 2006. Phylogenetic relatedness limits co-occurrence at fine spatial scales: evidence from the Schoenoid sedges (Cyperaceae: Schoeneae) of the Cape Floristic Region, South Africa. Am. Nat. 168(1):14-27.

SMITH, J. 2000. Nice work: but is it science? Nature. 408:293.

TILMAN, D. 2001. Functional diversity. In Encyclopedia of Biodiversity (S.A. Levin, ed.). Academic Press, San Diego, p. 109-120.

TILMAN, D., KNOPS, J., WEDIN, D., REICH, P., RITCHIE, M. \& SIEMANN, E. 1997. The influence of functional diversity and composition on ecosystem processes. Science. 277(5330):1300-1302.

TOFTS, R. \& SILVERTOWN, J. 2000. A phylogenetic approach to community assembly from a local species pool. P. Roy. Soc. B - Biol. Sci. 267(1441):363-369.

VILLÉGER, S., MASON, N.W.H. \& MOUILLOT, D. 2008. New multidimensional functional diversity indices for a multifaceted framework in functional ecology. Ecology. 89(8):2290-2301.

VALIENTE-BANUET, A. \& VERDÚ, M. 2007. Facilitation can increase the phylogenetic diversity of plant communities. Ecol. Lett. 10(11):1029-1036.

VAMOSI, S.M., HEARD, S.B., VAMOSI, J.C. \& WEBB, C.O. 2009. Emerging patterns in the comparative analysis of phylogenetic community structure. Mol. Ecol. 18(4):572-592.

VANE-WRIGHT, R.I., HUMPHRIES, C.J. \& WILLIAMS, P.M. 1991. What to protect: systematics and the agony of choice. Biol. Conserv. 55(2):235-254

VIOLLE, C., NAVAS, M.L., VILE, D., KAZAKOU, E., FORTUNEL, C., HUMMEL, I. \& GARNIER, E. 2007. Let the concept of trait be functional! Oikos. 116(5):882-892.

WAKE, D.B. \& LARSON, A. 1987. Multidimensional analysis of an evolving lineage. Science. 23(4823):42-48.

WALKER, B., KINZIG, A. \& LANGRIDGE, J. 1999. Plant attribute diversity, resilience, and ecosystem function: the nature and significance of dominant and minor species. Ecosystems. 2(1):95-113.

WARWICK, R.M. \& CLARKE, K.R. 1995. New "biodiversity" measures reveal a decrease in taxonomic distinctness with increasing stress. Mar. Ecol. Prog. Ser. 129:301-305. 
WARWICK, R.M. \& CLARKE, K.R. 1998. Taxonomic distinctness and environmental assessment. J. Appl. Ecol. 35(4):532-543.

WEBB, C.O. 2000. Exploring the phylogenetic structure of ecological communities: an example for rain forest trees. Am. Nat. 156(1):145-155.

WEBB, C.O., ACKERLY, D.D. \& KEMBEL, S.W. 2008 Phylocom: software for the analysis of community phylogenetic structure and trait evolution. Version 4.0.1. http://www.phylodiversity.net/phylocom/ (último acesso em 28/08/2009).

WEBB, C.O., ACKERLY, D.D., MCPEEK, M.A. \& DONOGHUE, M.J. 2002. Phylogenies and community ecology. Annu. Rev. Ecol. Syst. 33:475-505.

WEBB, C.O. \& DONOGHUE, M.J. 2005. Phylomatic: tree assembly for applied phylogenetics. Mol. Ecol. Not. 5(1):181-183.

WEIHER, E. \& KEDDY, P.A. 1995. Assembly rules, null models, and trait dispersion: new questions from old patterns. Oikos. 74(1):159-164.
WIKSTRÖM, N., SAVOLAINEN, V. \& CHASE, M.W. 2001. Evolution of the angiosperms: calibrating the family tree. P. Roy. Soc. B - Biol. Sci. 268(1482):2211-2220.

WILLIAMS, P.H., HUMPHRIES, C.J. \& VANE-WRIGHT, R.I. 1991. Measuring biodiversity: taxonomic relatedness for conservation priorities. Austr. Syst. Bot. 4(4):665-679.

WILSON, E.O. 1992. The diversity of life. Penguin, London, p.432.

WORSTER, D. 1994. Nature's economy: a history of ecological ideas. Cambridge University, Cambridge, p.423.

WRIGHT, S. 1982. Character change, speciation, and the higher taxa. Evolution. 36(3):427-443.

Recebido em 13/04/09 Versão Reformulada Recebida em 23/06/09 Publicado em 23/06/09 